\title{
Clinical Significance of Low Blood Testosterone Concentration in Men as a Cardiovascular Risk Factor From the Perspective of Blood Rheology
}

\author{
Takashi Hitsumoto
}

\begin{abstract}
Background: Recent clinical studies have indicated the importance of low blood testosterone concentration or whole blood passage time (WBPT) which reflects blood rheology as a cardiovascular risk factor. On the contrary, there are no reports regarding the association of blood testosterone concentrations and WBPT. This cross-sectional study aimed to elucidate the clinical significance of low blood testosterone concentration in men as a cardiovascular risk factor from the perspective of blood rheology using WBPT.
\end{abstract}

Methods: In total, 382 male patients with traditional cardiovascular risk factor and no history of cardiovascular disease (age (mean \pm standard deviation (SD)), $64 \pm 10$ years) were enrolled. Serum total testosterone concentration (T-T) was measured as a marker of testosterone level in vivo, and WBPT was also measured using microchannel array flow analyzer as a commercial device. The relationship between T-T and WBPT was evaluated.

Results: There was a significantly negative correlation between T-T and WBPT $(\mathrm{r}=-0.45 ; \mathrm{P}<0.001)$. Furthermore, multiple regression analysis revealed that T-T $(\beta=-0.25 ; \mathrm{P}<0.001)$ could be selected as an independent variable when WBPT was used as a subordinate factor. According to receiver operating characteristic curve analysis and the result of the previous report that determined WBPT of $>72.4 \mathrm{~s}$ as a risk for incidence of primary cardiovascular disease, T-T of $<551.4$ $\mathrm{ng} / \mathrm{dL}$ is the optimal cut-off point for discriminating high WBPT.

Conclusions: The study results showed that T-T is independently and inversely associated with WBPT in male patients with traditional cardiovascular risk factor and no history of cardiovascular disease. In addition, this study suggests that the incidence of primary cardiovascular events can be prevented by maintaining T-T at approximately $\geq$ $550 \mathrm{ng} / \mathrm{dL}$ from the perspective of blood rheology.

Keywords: Testosterone; Blood rheology; Cardiovascular risk factor; Skin autofluorescence; Oxidative stress; Men

Manuscript submitted March 15, 2019, accepted April 19, 2019

Hitsumoto Medical Clinic, 2-7-7, Takezakicyou, Shimonoseki City, Yamaguchi, 750-0025, Japan. Email: thitsu@jcom.home.ne.jp

doi: https://doi.org/10.14740/cr858

\section{Introduction}

In daily practice, reducing the incidence of cardiovascular event is primarily based on the management of traditional cardiovascular risk factors such as hypertension, dyslipidemia, diabetes mellitus, smoking habits, and obesity. Although these risk factors are important, they do not explain the incidence of every cardiovascular event [1-3]. Therefore, it is important to explore the novel risk factor of cardiovascular diseases to reduce the incidence of cardiovascular events. On the contrary, epidemiological and clinical studies reported that low blood testosterone concentration in men is associated with the incidence of cardiovascular disease [4-6]. Therefore, we should consider low blood testosterone concentration as a novel risk factor of cardiovascular disease in male patients.

Impairment of blood rheology is an important risk factor for cardiovascular disease as well as atherosclerosis $[7,8]$. In recent years, the microchannel array flow analyzer (MC-FAN) as a commercial device that assesses blood rheology by using microscopic images has been introduced in clinical practice [9]. MC-FAN has a simple methodology to evaluate blood rheology on the basis of whole blood passage time (WBPT), and it is superior to other methods with respect to the accuracy of channel dimensions and high reproducibility. In addition, some clinical studies have demonstrated relationships between increased WBPT and cardiovascular risk factors or the incidence of cardiovascular events [10-14].

Several studies exist regarding the relationships between testosterone and blood rheology [15-17]. However, no study has assessed the relationship between blood testosterone concentration and WBPT. Thus, this study aimed to elucidate the clinical significance of low blood testosterone concentration in men as a cardiovascular risk factor from the perspective of blood rheology using WBPT.

\section{Materials and Methods}

\section{Patients}

This cross-sectional study was performed at the Hitsumoto Medical Clinic in Yamaguchi, Japan, between September 2016 and August 2018. WBPT, the serum total testosterone concen- 
tration (T-T), and various clinical parameters were analyzed in 382 male patients with traditional cardiovascular risk factor (age (mean \pm standard deviation (SD)), $64 \pm 10$ years). Patients with a history of cardiovascular disease such as ischemic heart disease, stroke, peripheral artery disease, and heart failure admission were excluded from this study. This study was conducted in compliance with the ethical standards of the responsible institution on human subjects as well as the Helsinki Declaration. All patients provided informed consent, and the study protocol was approved by the Institutional Review Board of the Hitsumoto Medical Clinic (Approval number: 2016-07).

\section{Evaluation of blood rheology by MC-FAN}

The blood rheology was evaluated by measuring WBPT with an MC-FAN HR300 rheometer (MC Healthcare, Tokyo, Japan), as previously reported $[9,11]$. Briefly, the microchannel passage time for $100 \mu \mathrm{L}$ of physiological saline used as a control was measured; next, the microchannel passage time for $100 \mu \mathrm{L}$ of a heparinized whole blood sample obtained from a patient. The WBPT for patients was corrected for the passage time of physiological saline. The microchannel was $7-\mu \mathrm{m}$ wide, $30-\mu \mathrm{m}$ long, and $4.5-\mu \mathrm{m}$ deep. WBPT was measured within $60 \mathrm{~min}$ of blood sampling. The inter- and intra-assay coefficients of variation for WBPT were $8 \%$ and $5 \%$, respectively, indicating that the values of WBPT were within the acceptable range of reproducibility.

\section{Evaluation of cardiovascular risk factors}

The degree of obesity was assessed using body mass index, calculated as the ratio of weight $(\mathrm{kg})$ to the square of height $\left(\mathrm{m}^{2}\right)$. A current smoker was defined as an individual who smoked at least one cigarette per day in the last 28 days. Right brachial blood pressure was measured twice by using a mercury sphygmomanometer with the individual in sitting position. An average of two readings was used to determine systolic and diastolic blood pressures. Hypertension was defined as systolic blood pressure of $\geq 140 \mathrm{~mm} \mathrm{Hg}$, diastolic blood pressure of $\geq 90 \mathrm{~mm} \mathrm{Hg}$, or use of antihypertensive medication. Dyslipidemia was defined as low-density lipoprotein cholesterol level of $\geq 140 \mathrm{mg} / \mathrm{dL}$, high-density lipoprotein cholesterol level of $\leq 40 \mathrm{mg} / \mathrm{dL}$, a triglyceride level of $\geq 150$ $\mathrm{mg} / \mathrm{dL}$, or the use of antidyslipidemic medications. Diabetes mellitus was defined as having fasting blood glucose levels of $\geq 126 \mathrm{mg} / \mathrm{dL}$ or hemoglobin A1c levels of $\geq 6.5 \%$, which is estimated by the National Glycohemoglobin Standardization Program, or taking of anti-diabetic treatment. Skin autofluorescence (AF), reflecting the accumulation of advanced glycation end-products (AGEs) in tissues, was measured on the volar side of the forearm by using a commercial instrument (AGE Reader; DiagnOptics, Groningen, The Netherlands), as previously described [18]. Blood samples were harvested from the antecubital vein in the morning after $12 \mathrm{~h}$ of fasting. The levels of serum lipid, plasma glucose, insulin, and hemoglobin A1c, blood cell count, reactive oxygen metabo- lites (d-ROMs) test as a marker of oxidative stress, and T-T were subsequently measured. Total cholesterol, triglyceride, and high-density lipoprotein cholesterol concentrations were measured using the standard enzymatic methods. Low-density lipoprotein cholesterol concentration was determined using the Friedewald formula [19]. Patients with a serum triglyceride concentration of $\geq 400 \mathrm{mg} / \mathrm{dL}$ were excluded because the method is accurate only below this concentration. Plasma glucose and insulin levels were measured using the hexokinase ultraviolet absorption spectrophotometry method and chemiluminescence immunoassay, respectively. To measure insulin resistance, the homeostatic model assessment of insulin resistance (HOMA-IR) was used as follows [20]: HOMA-IR $=$ fasting glucose concentration $(\mathrm{mg} / \mathrm{dL}) \times$ fasting immunoreactive insulin concentration $(\mu \mathrm{g} / \mathrm{ml}) / 405$. The d-ROMs test, which reflects blood hydroperoxide concentrations, was performed using a commercial kit (Diacron, Grosseto, Italy) [21]. T-T was measured using a commercial kit (ARCHITECT Testosterone II, Abbott Japan, Tokyo).

\section{Statistical analysis}

The data were analyzed using Stat View-J 5.0 (HULINKS, Tokyo, Japan) and MedCalc for Windows version 14.8.1 (MedCalc Software, Ostend, Belgium). Continuous variables were expressed as mean $\pm \mathrm{SD}$. Between-group comparisons were performed using the Student's $t$-test or the Mann-Whitney Utest. The correlation coefficient was determined by using the Spearman's rank correlation analysis. Multivariate analysis was performed using multiple regression analysis. Receiver operating characteristic (ROC) curves were constructed, and the maximum Youden's index [22] was used to determine the optimal cut-off point of T-T for high WBPT. A P-value of $<$ 0.05 was considered to indicate statistical significance.

\section{Results}

Baseline clinical characteristics are summarized in Table 1. The mean value of T-T was $567.9 \pm 189.0$ (range: 39.0 1,234.7) $\mathrm{ng} / \mathrm{dL}$, and the mean value of WBPT was $57.5 \pm 15.9$ (range: 30.4 - 116.0) s. T-T and WBPT showed nearly normal distribution. The association between T-T and WBPT is shown in Figure 1. There was a significantly negatively correlation between these clinical parameters. Table 2 shows the association between WBPT or T-T and various clinical parameters. WBPT is significantly positively correlated with body mass index, smoking status, triglyceride levels, diabetes mellitus, HOMA-IR, skin AF, hematocrit, and d-ROMs test. WBPT is also significantly negatively correlated with high-density lipoprotein cholesterol levels. T-T is significantly negatively correlated with body mass index, serum triglyceride levels, diabetes mellitus, fasting blood glucose levels, HOMA-IR, hemoglobin A1c levels, skin AF, and d-ROMs test. T-T is also significantly positively correlated with high-density lipoprotein cholesterol levels. Table 3 shows the result of multiple regression analysis for WBPT or T-T as a subordinate factor. 
Table 1. Patients Characteristics

\begin{tabular}{|c|c|}
\hline $\mathrm{N}$ & 382 \\
\hline Age (years) & $64 \pm 10$ \\
\hline Body mass index $\left(\mathrm{kg} / \mathrm{m}^{2}\right)$ & $23.6 \pm 3.8$ \\
\hline Current smoker; n (\%) & $127(33)$ \\
\hline Hypertension; n (\%) & $289(76)$ \\
\hline Systolic BP (mm Hg) & $141 \pm 18$ \\
\hline Diastolic BP (mm Hg) & $89 \pm 24$ \\
\hline Dyslipidemia; n (\%) & $197(52)$ \\
\hline Total cholesterol (mg/dL) & $212 \pm 42$ \\
\hline LDL cholesterol (mg/dL) & $139 \pm 38$ \\
\hline Triglyceride (mg/dL) & $138 \pm 69$ \\
\hline HDL cholesterol (mg/dL) & $45 \pm 14$ \\
\hline Diabetes mellitus; n (\%) & $123(32)$ \\
\hline FBG (mg/dL) & $110 \pm 14$ \\
\hline HOMA-IR & $2.2 \pm 1.2$ \\
\hline HbAlc (\%) & $6.2 \pm 1.0$ \\
\hline Skin AF (arbitrary units) & $2.7 \pm 0.5$ \\
\hline White blood cell $(/ \mu \mathrm{L})$ & $6,116 \pm 1,563$ \\
\hline Hematocrit (\%) & $42.3 \pm 4.1$ \\
\hline Platelet $\left(10^{4} / \mu \mathrm{L}\right)$ & $24 \pm 6$ \\
\hline d-ROMs test (U. Carr) & $320 \pm 96$ \\
\hline $\mathrm{T}-\mathrm{T}(\mathrm{ng} / \mathrm{dL})$ & $567.9 \pm 189.0$ \\
\hline WBPT (s) & $57.5 \pm 15.9$ \\
\hline \multicolumn{2}{|l|}{ Medications } \\
\hline RAS inhibitor; n (\%) & $142(37)$ \\
\hline Statin; n $(\%)$ & $120(31)$ \\
\hline Anti-diabetic drugs; n (\%) & $84(22)$ \\
\hline
\end{tabular}

Data are expressed mean \pm SD. BP: blood pressure; LDL: low-density lipoprotein; HDL: high-density lipoprotein; FBG: fasting blood glucose; HOMA-IR: homeostatic model assessment of insulin resistance; $\mathrm{HbA1c}$ : hemoglobin A1c; AF: autofluorescence; d-ROMs: derivatives of reactive oxygen metabolites; T-T: total testosterone; WBPT: whole blood passage time; RAS: renin-angiotensin system.

Explanatory factors were selected either by checking the multicollinearity among variables or by the stepwise method. Skin AF, T-T, body mass index, d-ROMs test, and hematocrit were selected as independent variables when WBPT was used as a subordinate factor. However, WBPT, HOMA-IR, skin AF, and $\mathrm{d}$-ROMs test were selected as independent variables when using T-T as a subordinate factor. Figure 2 shows the ROC curve analysis for the detection of high WBPT of $>72.4 \mathrm{~s}$ based on a previous report to predict primary cardiovascular events [13]. Maximum Youden's index indicated that a T-T of $<551.4 \mathrm{ng} /$ dL was the optimal cut-off point to determine WBPT as $>72.4$ s, with sensitivity of $88.5 \%$ and specificity of $61.7 \%$. Table 4 shows the comparisons of clinical parameters of the high T-T $(\geq 551.4 \mathrm{ng} / \mathrm{dL})$ and low T-T $(<551.4 \mathrm{ng} / \mathrm{dL})$ patients. In addition, body mass index, presence of diabetes mellitus, fasting

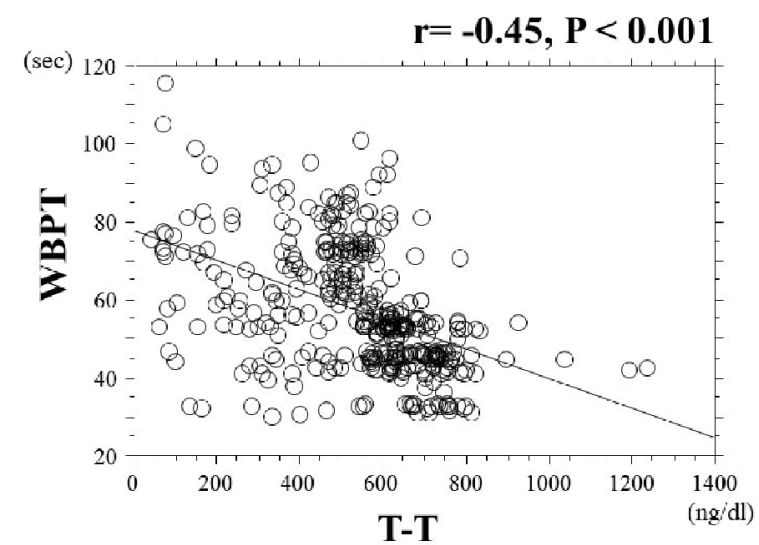

Figure 1. The association between T-T and WBPT. There was a significantly negative correlation between T-T and WBPT $(r=-0.45, \mathrm{P}<$ 0.001). T-T: total testosterone; WBPT: whole blood passage time.

blood glucose levels, HOMA-IR, hemoglobin A1c levels, skin AF, d-ROMs test, and WBPT were considerably higher, and the high-density lipoprotein cholesterol concentrations were considerably lower in patients with low T-T than in those with high T-T.

\section{Discussion}

In the current study, there was a significantly negative correlation between T-T and WBPT. Furthermore, multiple regression analysis indicated that these two biomarkers had independent association with each other after adjustment of related factors. In addition, ROC curve analysis clarified that T-T cut-off at $<$ $551.4 \mathrm{ng} / \mathrm{dL}$ was an optimal cut-off point for discriminating high WBPT of $>72.4 \mathrm{~s}$ which was reported as the predicted value of primary cardiovascular events. The results of the current study also indicated that d-ROMs test as a marker of oxidative stress and skin AF as a marker of AGEs in tissues were selected as independent variables for both WBPT and T-T as subordinate factors.

WBPT is known to be reflecting characters of whole blood sample; WBPT affects several factors such as leukocyte adhesive capacity, percentage of red blood cells, red blood cell deformity, platelet aggregation, and plasma viscosity. The study results indicate that hematocrit levels which reflect percentage of red blood cell are independently associated with WBPT, which is consistent with previous reports $[12,14,23]$. On the contrary, researchers reported that testosterone improves blood rheology by several mechanisms such as erythrocyte membrane lipid composition, platelet activation and reactivity, and blood viscosity [15-17]. In addition, Damber et al reported that decreased secretion of testosterone from the testis primarily owing to reduced blood flow in an animal model [24], suggesting that impairment of blood rheology in the testis may decrease testosterone production. Thus, results of previous and this study indicate that testosterone and blood rheology are associated with each other, and consequently, impairment in blood rheology may cause primary cardiovascular events 
Table 2. Relationships Between WBPT, T-T and Various Clinical Parameters

\begin{tabular}{|c|c|c|}
\hline & \multirow{2}{*}{$\begin{array}{l}\text { WBPT } \\
\mathbf{r}\end{array}$} & \multirow{2}{*}{$\begin{array}{l}\text { T-T } \\
\mathbf{r}\end{array}$} \\
\hline & & \\
\hline Age & -0.03 & 0.03 \\
\hline Body mass index & $0.22 * * *$ & $-0.16^{* *}$ \\
\hline Current smoker $($ Yes $=1$, No $=0)$ & $0.18^{*}$ & -0.06 \\
\hline Hypertension $(\mathrm{Yes}=1, \mathrm{No}=0)$ & 0.05 & -0.07 \\
\hline Systolic BP & 0.09 & -0.07 \\
\hline Diastolic BP & 0.06 & -0.04 \\
\hline Dyslipidemia $($ Yes $=1$, No $=0)$ & 0.05 & 0.04 \\
\hline Total cholesterol & 0.04 & 0.03 \\
\hline LDL cholesterol & 0.04 & 0.04 \\
\hline Triglyceride & $0.14 * *$ & $-0.12 *$ \\
\hline HDL cholesterol & $-0.16^{* *}$ & $0.11 *$ \\
\hline Diabetes mellitus $(\mathrm{Yes}=1, \mathrm{No}=0)$ & $0.15 * *$ & $-0.12 *$ \\
\hline FBG & 0.07 & $-0.11 *$ \\
\hline HOMA-IR & $0.13 *$ & $-0.36 * * *$ \\
\hline $\mathrm{HbA1c}$ & 0.10 & $-0.12 *$ \\
\hline Skin AF & $0.49 * * *$ & $-0.31 * * *$ \\
\hline White blood cell & 0.09 & -0.03 \\
\hline Hematocrit & $0.24 * * *$ & 0.03 \\
\hline Platelet & 0.07 & 0.06 \\
\hline d-ROMs test & $0.28 * * *$ & $-0.25 * * *$ \\
\hline RAS inhibitor $($ Yes $=1$, No $=0$ ) & -0.08 & 0.05 \\
\hline Statin $($ Yes $=1$, No $=0)$ & -0.07 & 0.04 \\
\hline Anti-diabetic drugs $(\mathrm{Yes}=1, \mathrm{No}=0)$ & 0.09 & 0.07 \\
\hline
\end{tabular}

Data are expressed mean $\pm \mathrm{SD}$. ${ }^{*} \mathrm{P}<0.05$; ${ }^{* *} \mathrm{P}<0.01$; ${ }^{* * *} \mathrm{P}<0.001$. WBPT: whole blood passage time; T-T: total testosterone; BP: blood pressure; LDL: low-density lipoprotein; HDL: high-density lipoprotein; FBG: fasting blood glucose; HOMA-IR: homeostatic model assessment of insulin resistance; HbA1c: hemoglobin A1c; AF: autofluorescence; d-ROMs: derivatives of reactive oxygen metabolites; RAS: renin-angiotensin system.

in male patients with traditional cardiovascular risk factor. It is useful to know the target cut-off level of T-T for predicting abnormal WBPT in clinical settings. This study clarified the clinical usefulness of assessing T-T for detecting high WBPT of $>72.4 \mathrm{~s}$, which has been demonstrated to be a predictor of primary cardiovascular events. ROC curve analysis for WBPT as high as $>72.4 \mathrm{~s}$ indicated that a T-T of $<551.4 \mathrm{ng} / \mathrm{dl}$ clarified the optimal cut-off point for discriminating high WBPT. On the contrary, Ohlsson et al reported that high serum testosterone which is indicated by T-T of $>550 \mathrm{ng} / \mathrm{dL}$ predicted a reduced 5-year risk of cardiovascular events in elderly men, including $26.2 \%$ risk of history of cardiovascular disease [25]. In addition, this study suggests that the incidence of primary cardiovascular events in male patients with traditional cardiovascular risk factor can be prevented by maintaining T-T at approximately $\geq 550 \mathrm{ng} / \mathrm{dL}$ from the perspective of blood rhe-
Table 3. Multiple Regression Analysis

\begin{tabular}{|cll}
\hline Explanatory factor & $\boldsymbol{\beta}$ & P value \\
\hline (A) Skin AF & 0.32 & $<0.001$ \\
T-T & -0.25 & $<0.001$ \\
Body mass index & 0.17 & $<0.001$ \\
d-ROMs test & 0.14 & 0.005 \\
Hematocrit & 0.13 & 0.007 \\
Current smoker & 0.08 & 0.083 \\
(B) WBPT & -0.30 & $<0.001$ \\
HOMA-IR & -0.28 & $<0.001$ \\
Skin AF & -0.15 & 0.004 \\
d-ROMs test & -0.12 & 0.010 \\
Body mass index & -0.07 & 0.139 \\
\hline
\end{tabular}

(A) Subordinate factor is WBPT; $R^{2}=0.33 ; P<0.001$. (B) Subordinate factor is $\mathrm{T}-\mathrm{T} ; \mathrm{R}^{2}=0.30 ; \mathrm{P}<0.001$. AF: autofluorescence; $\mathrm{T}-\mathrm{T}$ : total testosterone; d-ROMs: derivatives of reactive oxygen metabolites; HOMAIR: homeostatic model assessment of insulin resistance; WBPT: whole blood passage time.

ology.

Increased oxidative stress is well known to play a crucial role in the incidence of cardiovascular disease. In addition, several studies have reported that oxidative stress could cause impairment of blood rheology [26, 27]. This study also indicated that d-ROMs test, an oxidative stress marker in vivo, could be selected as an independent factor for WBPT, thereby suggesting that increased oxidative stress is associated with impairment of blood rheology in male patients with traditional cardiovascular risk factor. On the contrary, the study results also indicated that increase of d-ROMs test was selected as an

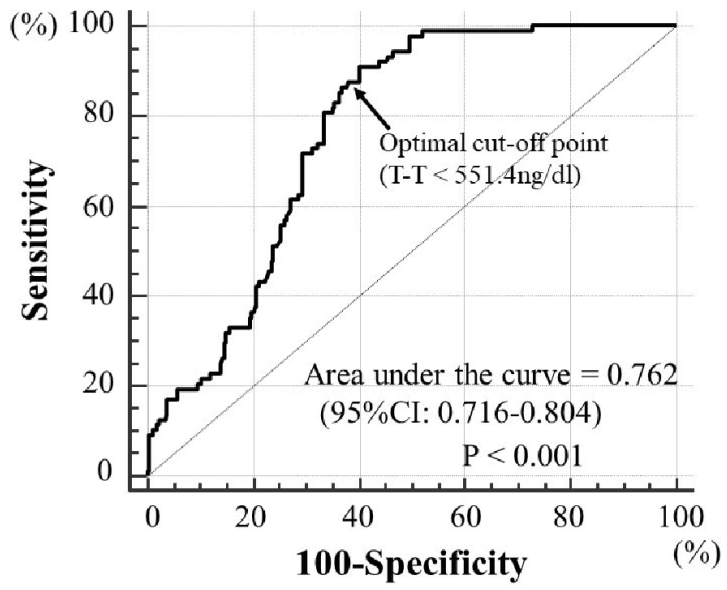

Figure 2. Receiver operating characteristic curve analysis for detection of high WBPT using T-T. Receiver operating characteristics determined the optimal cut-off point of T-T for high WBPT (> $72.4 \mathrm{~s}$ ). The area under curve was $0.762(P<0.001)$. Maximum Youden's index indicated that a T-T of $<551.4 \mathrm{ng} / \mathrm{dL}$ is an optimal cut-off point to determine high WBPT, with sensitivity of $88.5 \%$ and specificity of $61.7 \%$. Arrow indicates the optimal cut-off point. T-T: total testosterone; WBPT: whole blood passage time; $\mathrm{Cl}$ : confidence interval. 
Table 4. Comparisons of Clinical Parameters of the High T-T and Low T-T Patients

\begin{tabular}{|c|c|c|c|}
\hline & \multicolumn{2}{|c|}{ T-T (ng/dL) } & \multirow{2}{*}{ P value } \\
\hline & High T-T $(n=232) \geq 551.4$ & Low T-T $(n=150)<551.4$ & \\
\hline Age (years) & $64 \pm 11$ & $64 \pm 10$ & 0.573 \\
\hline Current smoker; n (\%) & $75(32)$ & $52(35)$ & 0.673 \\
\hline Hypertension; n (\%) & $172(74)$ & $117(78)$ & 0.424 \\
\hline Diastolic BP (mm Hg) & $88 \pm 22$ & $90 \pm 26$ & 0.231 \\
\hline Dyslipidemia; n (\%) & $121(52)$ & $76(51)$ & 0.776 \\
\hline Total cholesterol (mg/dL) & $213 \pm 41$ & $210 \pm 44$ & 0.431 \\
\hline LDL cholesterol (mg/dL) & $140 \pm 37$ & $138 \pm 38$ & 0.534 \\
\hline Triglyceride (mg/dL) & $136 \pm 64$ & $148 \pm 77$ & 0.078 \\
\hline HOMA-IR & $1.9 \pm 1.1$ & $2.6 \pm 1.2$ & $<0.001$ \\
\hline $\mathrm{HbA1c}(\%)$ & $6.1 \pm 1.0$ & $6.4 \pm 1.1$ & 0.025 \\
\hline Skin AF (arbitrary units) & $2.6 \pm 0.5$ & $2.9 \pm 0.5$ & $<0.001$ \\
\hline White blood cell $(/ \mu \mathrm{L})$ & $6,080 \pm 1,524$ & $6,171 \pm 1,624$ & 0.583 \\
\hline Hematocrit (\%) & $42.3 \pm 4.1$ & $42.2 \pm 4.1$ & 0.894 \\
\hline Platelet $\left(10^{4} / \mu \mathrm{L}\right)$ & $24 \pm 6$ & $24 \pm 6$ & 0.956 \\
\hline d-ROMs test (U. Carr) & $305 \pm 94$ & $342 \pm 95$ & $<0.001$ \\
\hline WBPT (s) & $51.8 \pm 12.9$ & $66.2 \pm 16.2$ & $<0.001$ \\
\hline
\end{tabular}

Data are expressed mean \pm SD. T-T: total testosterone; BP: blood pressure; LDL: low-density lipoprotein; HDL: high- density lipoprotein; FBG: fasting blood glucose; HOMA-IR: homeostatic model assessment of insulin resistance; HbA1c: hemoglobin A1c; AF: autofluorescence; d-ROMs: derivatives of reactive oxygen metabolites; WBPT: whole blood passage time; RAS: renin-angiotensin system.

independent variable for low T-T. Previous studies also reported the inverse association between oxidative stress and testosterone [28-30]. Thus, the results of this and previous studies indicated that oxidative stress is an important target factor for both blood rheology and testosterone.

Numerous studies exist regarding the relations of AGEs and various health problems. Furthermore, researchers emphasized the importance of skin AF as a cardiovascular risk factor [31-33]. Skin AF is evaluated based on the autofluorescence levels on the volar side of the lower arm, and it is reported to be indicating the levels of AGEs such as pentosidine, N(epsilon)(carboxymethyl)lysine, and N(epsilon)-(carboxyethyl)lysine according to the results of skin biopsy [34]. Conversely, hemorheology, assessed by MC-FAN, is an in vitro measurement that uses artificial blood vessels, with a 7- $\mu \mathrm{m}$ wide, 30- $\mu \mathrm{m}$ long, and 4.5- $\mu \mathrm{m}$ deep vessel lumen. Thus, the assessment of hemorheology by MC-FAN is assumed to correspond to values which are obtained for small vessels. Small vessels are considered to be present not only in a portion of the volar side of the lower arm but also in systemic organs. Therefore, independent association between skin AF and WBPT in this study were considered as to be indicating the close relation of AGEs and impairment of blood rheology in systemic organs, including heart and brain. Several basic studies have indicated that AGEs influence hemorheology by mechanisms such as platelet aggregation, leukocyte-endothelial interaction, and morphological changes in the erythrocyte membrane [35-37]. The study results also indicated the importance of AGEs on blood rheology in male patients with traditional cardiovascular risk factor. In addition, skin AF was independently inversely associated with T-T in this study. Several reports demonstrated the association between AGEs and testosterone [38, 39]. Zhao et al using animal models reported that AGEs inhibit testosterone secretion [38]. On the contrary, Xie et al reported that physiological testosterone prevented AGE-induced injury in human endothelial cells [39]. Thus, the results of this and pre- 
vious studies indicated that testosterone and AGEs were associated with each other, and consequently, they could cause the incidence of cardiovascular events in male patients. Isami et al reported that skin AF levels were associated with habitual lifestyle behaviors, such as physical activity, nonsmoking, adequate sleep, low mental stress level, eating breakfast, and abstaining from sugary food [40]. In addition, other reports also indicated that WBPT, T-T, and d-ROMs test had significant association with life style or metabolic syndrome [11, 41, 42]. Therefore, maintaining a good life style is recommended in male patients with traditional cardiovascular risk factor from the perspective of biomarkers such as skin AF, WBPT, T-T, and d-ROMs test.

\section{Limitations}

This study has several limitations. First, the medical treatments for traditional cardiovascular risk factor such as hypertension, dyslipidemia, and anti-diabetic medications might have influenced the study results. Second, angiography, computed tomography, magnetic resonance imaging, and echocardiography were not completely performed for all patients. Therefore, asymptomatic cardiovascular disease might have been undetected. Third, parameters of testosterone such as free testosterone, sex hormone binding globulin, or blood estradiol concentrations were not measured in this study. Finally, this was a cross-sectional study conducted in a single unit with a relatively small sample size. Additional prospective studies, including interventional therapy evaluation, are required to assess the validity of this study's results.

\section{Conclusions}

In conclusion, the results of this study showed that T-T is independently inversely associated with WBPT in patients with traditional cardiovascular risk factor and no history of cardiovascular disease. In addition, this study suggests that the incidence of primary cardiovascular events can be prevented by maintaining T-T at approximately $\geq 550 \mathrm{ng} / \mathrm{dL}$ from the perspective of blood rheology.

\section{Acknowledgments}

The author is grateful to the individuals who participated in this study.

\section{Financial Disclosure}

None to declare.

\section{Conflict of Interest}

None to declare.

\section{Informed Consent}

All patients provided informed consent.

\section{Author Contributions}

The author was involved in preparing the study design as well as acquisition, analysis, and interpretation of data.

\section{References}

1. Takashima N, Ohkubo T, Miura K, Okamura T, Murakami Y, Fujiyoshi A, Nagasawa SY, et al. Long-term risk of BP values above normal for cardiovascular mortality: a 24-year observation of Japanese aged 30 to 92 years. J Hypertens. 2012;30(12):2299-2306.

2. Alagona P, Jr., Ahmad TA. Cardiovascular disease risk assessment and prevention: current guidelines and limitations. Med Clin North Am. 2015;99(4):711-731.

3. Intensive blood-glucose control with sulphonylureas or insulin compared with conventional treatment and risk of complications in patients with type 2 diabetes (UKPDS 33). UK Prospective Diabetes Study (UKPDS) Group. Lancet. 1998;352(9131):837-853.

4. Laughlin GA, Barrett-Connor E, Bergstrom J. Low serum testosterone and mortality in older men. J Clin Endocrinol Metab. 2008;93(1):68-75.

5. Akishita M, Hashimoto M, Ohike Y, Ogawa S, Iijima K, Eto M, Ouchi Y. Low testosterone level as a predictor of cardiovascular events in Japanese men with coronary risk factors. Atherosclerosis. 2010;210(1):232-236.

6. Pastuszak AW, Kohn TP, Estis J, Lipshultz LI. Low plasma testosterone is associated with elevated cardiovascular disease biomarkers. J Sex Med. 2017;14(9):1095-1103.

7. Tzoulaki I, Murray GD, Lee AJ, Rumley A, Lowe GD, Fowkes FG. Relative value of inflammatory, hemostatic, and rheological factors for incident myocardial infarction and stroke: the Edinburgh Artery Study. Circulation. 2007;115(16):2119-2127.

8. Cowan AQ, Cho DJ, Rosenson RS. Importance of blood rheology in the pathophysiology of atherothrombosis. Cardiovasc Drugs Ther. 2012;26(4):339-348.

9. Kikuchi Y, Sato K, Mizuguchi Y. Modified cell-flow microchannels in a single-crystal silicon substrate and flow behavior of blood cells. Microvasc Res. 1994;47(1):126139.

10. Kotani K, Satoh N, Yamada K, Taniguchi N, Shimatsu A. The influence of metabolic syndrome and chronic kidney disease on hemorheology assessed by the microchannel method. J Physiol Anthropol. 2010;29(5):157-160.

11. Hitsumoto T. Factors affecting impairment of blood rheology in obese subjects. J Cardiol. 2012;60(5):401-406.

12. Yoshida K, Kimura T, Aoki T, Tsunekawa K, Araki O, Shoho Y, Nara M, et al. Fasting serum insulin levels and insulin resistance are associated with blood rheology in Japanese young adults without diabetes. J Int Med Res. 
2016;44(3):496-507.

13. Hitsumoto T. Usefulness of the whole blood passage time as a predictor of primary cardiovascular events in patients with traditional cardiovascular risk factors. Cardiol Res. 2018;9(4):231-238.

14. Hitsumoto T. Clinical impact of hemorheology on subclinical myocardial injury in patients with hypertension. J Clin Med Res. 2018;10(12):928-935.

15. Angelova P, Momchilova A, Petkova D, Staneva G, Pankov R, Kamenov Z. Testosterone replacement therapy improves erythrocyte membrane lipid composition in hypogonadal men. Aging Male. 2012;15(3):173-179.

16. Karolczak K, Konieczna L, Kostka T, Witas PJ, Soltysik B, Baczek T, Watala C. Testosterone and dihydrotestosterone reduce platelet activation and reactivity in older men and women. Aging (Albany NY). 2018;10(5):902-929.

17. Zhao C, Moon du G, Park JK. Effect of testosterone undecanoate on hematological profiles, blood lipid and viscosity and plasma testosterone level in castrated rabbits. Can Urol Assoc J. 2013;7(3-4):E221-225.

18. Meerwaldt R, Links TP, Graaff R, Hoogenberg K, Lefrandt JD, Baynes JW, Gans RO, et al. Increased accumulation of skin advanced glycation end-products precedes and correlates with clinical manifestation of diabetic neuropathy. Diabetologia. 2005;48(8):1637-1644.

19. Friedewald WT, Levy RI, Fredrickson DS. Estimation of the concentration of low-density lipoprotein cholesterol in plasma, without use of the preparative ultracentrifuge. Clin Chem. 1972;18(6):499-502.

20. Matthews DR, Hosker JP, Rudenski AS, Naylor BA, Treacher DF, Turner RC. Homeostasis model assessment: insulin resistance and beta-cell function from fasting plasma glucose and insulin concentrations in man. Diabetologia. 1985;28(7):412-419.

21. Cesarone MR, Belcaro G, Carratelli M, Cornelli U, De Sanctis MT, Incandela L, Barsotti A, et al. A simple test to monitor oxidative stress. Int Angiol. 1999;18(2):127-130.

22. Schisterman EF, Perkins NJ, Liu A, Bondell H. Optimal cut-point and its corresponding Youden Index to discriminate individuals using pooled blood samples. Epidemiology. $2005 ; 16(1): 73-81$.

23. Hitsumoto T. Association of hemorheology with highsensitivity cardiac troponin $\mathrm{T}$ levels in patients with type 2 diabetes mellitus assessed by microchannel array flow analyzer. Cardiol Res. 2017;8(6):304-311.

24. Damber JE, Bergh A, Daehlin L. Testicular blood flow, vascular permeability, and testosterone production after stimulation of unilaterally cryptorchid adult rats with human chorionic gonadotropin. Endocrinology. 1985;117(5):1906-1913.

25. Ohlsson C, Barrett-Connor E, Bhasin S, Orwoll E, Labrie F, Karlsson MK, Ljunggren $\mathrm{O}$, et al. High serum testosterone is associated with reduced risk of cardiovascular events in elderly men. The MrOS (Osteoporotic Fractures in Men) study in Sweden. J Am Coll Cardiol. 2011;58(16):1674-1681.

26. Tzoulaki I, Murray GD, Lee AJ, Rumley A, Lowe GD, Fowkes FG. Inflammatory, haemostatic, and rheological markers for incident peripheral arterial disease: Edin- burgh Artery Study. Eur Heart J. 2007;28(3):354-362.

27. Podrez EA, Byzova TV, Febbraio M, Salomon RG, Ma Y, Valiyaveettil M, Poliakov E, et al. Platelet CD36 links hyperlipidemia, oxidant stress and a prothrombotic phenotype. Nat Med. 2007;13(9):1086-1095.

28. Haring R, Baumeister SE, Volzke H, Dorr M, Kocher T, Nauck M, Wallaschofski H. Prospective inverse associations of sex hormone concentrations in men with biomarkers of inflammation and oxidative stress. J Androl. 2012;33(5):944-950.

29. Rovira-Llopis S, Banuls C, de Maranon AM, Diaz-Morales N, Jover A, Garzon S, Rocha M, et al. Low testosterone levels are related to oxidative stress, mitochondrial dysfunction and altered subclinical atherosclerotic markers in type 2 diabetic male patients. Free Radic Biol Med. 2017;108:155-162.

30. Hwang TI, Liao TL, Lin JF, Lin YC, Lee SY, Lai YC, Kao SH. Low-dose testosterone treatment decreases oxidative damage in TM3 Leydig cells. Asian J Androl. 2011;13(3):432-437.

31. Hangai M, Takebe N, Honma H, Sasaki A, Chida A, Nakano R, Togashi $\mathrm{H}$, et al. Association of advanced glycation end products with coronary artery calcification in japanese subjects with type 2 diabetes as assessed by skin autofluorescence. J Atheroscler Thromb. 2016;23(10):11781187.

32. Hitsumoto T. Clinical significance of skin autofluorescence in patients with type 2 diabetes mellitus with chronic heart failure. Cardiol Res. 2018;9(2):83-89.

33. Yamagishi S, Fukami K, Matsui T. Evaluation of tissue accumulation levels of advanced glycation end products by skin autofluorescence: A novel marker of vascular complications in high-risk patients for cardiovascular disease. Int J Cardiol. 2015;185:263-268.

34. Meerwaldt R, Graaff R, Oomen PHN, Links TP, Jager JJ, Alderson NL, Thorpe SR, et al. Simple non-invasive assessment of advanced glycation endproduct accumulation. Diabetologia. 2004;47(7):1324-1330.

35. Hasegawa Y, Suehiro A, Higasa S, Namba M, Kakishita E. Enhancing effect of advanced glycation end products on serotonin-induced platelet aggregation in patients with diabetes mellitus. Thromb Res. 2002;107(6):319-323.

36. Morigi M, Angioletti S, Imberti B, Donadelli R, Micheletti G, Figliuzzi M, Remuzzi A, et al. Leukocyte-endothelial interaction is augmented by high glucose concentrations and hyperglycemia in a NF-kB-dependent fashion. J Clin Invest. 1998;101(9):1905-1915.

37. Awasthi S, Gayathiri SK, Ramya R, Duraichelvan R, Dhason A, Saraswathi NT. Advanced glycation-modified human serum albumin evokes alterations in membrane and eryptosis in erythrocytes. Appl Biochem Biotechnol. 2015;177(5):1013-1024.

38. Zhao YT, Qi YW, Hu CY, Chen SH, Liu Y. Advanced glycation end products inhibit testosterone secretion by rat Leydig cells by inducing oxidative stress and endoplasmic reticulum stress. Int J Mol Med. 2016;38(2):659-665.

39. Xie Y, Yu D, Wu J, Li L. Protective effects of physiological testosterone on advanced glycation end productinduced injury in human endothelial cells. Mol Med Rep. 
2017;15(3):1165-1171.

40. Isami F, West BJ, Nakajima S, Yamagishi SI. Association of advanced glycation end products, evaluated by skin autofluorescence, with lifestyle habits in a general Japanese population. J Int Med Res. 2018;46(3):1043-1051.

41. Tanabe M, Akehi Y, Nomiyama T, Murakami J, Yanase

T. Total testosterone is the most valuable indicator of metabolic syndrome among various testosterone values in middle-aged Japanese men. Endocr J. 2015;62(2):123132.

42. Kotani K, Tsuzaki K, Sakane N, Taniguchi N. The correlation between small dense LDL and reactive oxygen metabolites in a physical activity intervention in hyperlipidemic subjects. J Clin Med Res. 2012;4(3):161-166. 